Quim. Nova, Vol. 35, No. 8, 1582-1586, 2012

\title{
IDENTIFICAÇÃO DOS COMPOSTOS DEFENSIVOS ENCONTRADOS NAS GLÂNDULAS METATORÁCICA E ABDOMINAIS DORSAIS DOS PERCEVEJOS Loxa deducta E Pellaea stictica (Heteroptera: Pentatomidae)
}

\author{
Carla F. Fávaro e Paulo H. G. Zarbin* \\ Departamento de Química, Universidade Federal do Paraná, CP 19081, 81531-990 Curitiba - PR, Brasil
}

Recebido em 18/1/12; aceito em 23/4/12; publicado na web em 27/7/12

\begin{abstract}
IDENTIFICATION OF THE DEFENSIVE COMPOUNDS IN METATHORACIC AND DORSAL ABDOMINAL GLANDS OF THE STINK BUGS Loxa deducta AND Pellaea stictica (Heteroptera: Pentatomidae). The dorsal abdominal glands (DAGs) contents in nymphs and the metathoracic glands (MTG) in adult males and females (10, 20 and 30 days old) were characterized and quantified for Loxa deducta and Pellaea stictica. The major component for nymphs and adults was tridecane, with lesser amounts of other aliphatic hydrocarbons, aldehydes, oxo-alkenals and esters. The five nymphal instars showed significant differences in compounds proportion. No significant differences was detected in MTG components proportion between sexes. However (E)-2-hexenal and (E)-2-decenyl acetate was significantly lower in adults from 10 to 20 days.
\end{abstract}

Keywords: allomone; pheromone; defensive compounds.

\section{INTRODUÇÃO}

Os pentatomídeos são bem conhecidos pela produção de odores desagradáveis que têm dupla finalidade, de defesa contra a predação e feromônio de alarme. ${ }^{1}$ Estes odores defensivos são produzidos em dois diferentes tipos de glândulas, dependendo do estágio de vida destes insetos. Nos adultos, são produzidos em uma glândula grande, bem definida e de cor laranja, localizada na região metatorácica do inseto (MTG), enquanto as ninfas os produzem nas chamadas glândulas abdominais dorsais (DAGs). ${ }^{1}$

O principal método de extração dos componentes feromonais presentes na MTG é através da extração direta do conteúdo da glândula. No entanto, a extração dos componentes das DAGs é feita a partir de extratos das exúvias liberadas no processo de troca de instar, pois as ninfas de pentatomídeos transferem o conteúdo das DAGs para suas exúvias e é possível obtê-los quando a extração é realizada em um período menor que $24 \mathrm{~h}$ após a muda. ${ }^{2}$

Estudos anteriores sobre a química defensiva em pentatomídeos mostram quem estes compostos com características defensivas fazem parte de grupos como aldeídos insaturados, hidrocarbonetos alifáticos saturados e insaturados; oxo-alcenais e ésteres. ${ }^{3}$ Nesses trabalhos, são descritas diferenças qualitativas e quantitativas na composição da mistura defensiva entre ninfas de diferentes instares, ${ }^{2,4}$ entre ninfas e adultos ${ }^{5}$ e entre adultos machos e fêmeas. ${ }^{6}$ Entretanto, estudos relacionando a composição da mistura feromonal defensiva com o aumento da idade do inseto adulto foram relatados para o inseto Leptoglossus phyllopus (Heteroptera: Coreidae) ${ }^{7}$ e, recentemente, nosso grupo relatou para o percevejo Pallantia macunaima. ${ }^{8}$

Este trabalho descreve a identificação, comparação e quantificação dos compostos encontrados nas exúvias de ninfas dos cinco instares e nas glândulas metatorácicas em machos e fêmeas com 10, 20 e 30 dias de idade adulta de duas espécies de percevejos, Loxa deducta Walker e Pellaea stictica (Dallas).

\section{PARTE EXPERIMENTAL}

\section{Insetos}

Exemplares de adultos das duas espécies foram enviados pelo Laboratório de Bioecologia de Percevejos da Embrapa Soja, de Londrina. Foram separados por espécies e colocados em gaiolas plásticas mantidas em câmaras BOD a $25^{\circ} \mathrm{C}$ com fotoperíodo de 14 h de luz e umidade relativa de $70 \%$. Como sugerido por Siqueira, ${ }^{9}$ os insetos foram alimentados com vagens verdes de feijão, Phaseolus vulgaris (L.), sementes de soja, Glycine max (L.), amendoim, Arachis hypogaea (L.), e frutos de ligustro, Ligustrum lucidum Ait.

\section{Extração do conteúdo das glândulas abdominais dorsais (DAGs)}

O desenvolvimento das ninfas e liberação das exúvias foi acompanhado diariamente desde a eclosão dos ovos até se tornarem adultas. Os extratos foram feitos com hexano grau HPLC bidestilado, respeitando o número de 10 exúvias de primeiro instar por extrato, 8 exúvias de segundo instar, 6 exúvias de terceiro instar, 4 exúvias de quarto instar e 2 exúvias de quinto instar. As exúvias foram mantidas em hexano durante o período de 24 h sendo, logo após, retiradas e os extratos acondicionados em freezer para análises posteriores. ${ }^{2,8}$

\section{Extração do conteúdo da glândula metatorácica (MTG)}

Um adulto foi depositado com a parte dorsal voltada para cima em placa de Petri. O processo de dissecação consistiu em cortar as arestas abdominais dorsais da cutícula do inseto até a região metatorácica. A cutícula foi puxada para trás e as vísceras removidas. A glândula foi retirada e imersa em $200 \mu \mathrm{L}$ de hexano grau HPLC bidestilado. Após 24 h, a glândula foi retirada e o extrato armazenado a $-20{ }^{\circ} \mathrm{C} .{ }^{8}$

\section{Identificação dos compostos}

Os extratos foram analisados ( $1 \mu \mathrm{L}$ de extrato) em cromatógrafo a gás (CG), modelo Shimadzu QP-2010, equipado com detector de ionização de chama (FID) e com coluna capilar DB-5 (30 m × 0,25 mm 
D.I. $\times 0,25 \mu \mathrm{m}$ de filme) (J \& W Scientific, Folsom, Califórnia, EUA). O CG foi operado em modo splitless $\left(250^{\circ} \mathrm{C}\right)$, com programação de temperatura de $50^{\circ} \mathrm{C}$ por $1 \mathrm{~min}$, aumentando $7^{\circ} \mathrm{C} / \mathrm{min}$ até $250{ }^{\circ} \mathrm{C}$ e permanecendo nesta temperatura por $10 \mathrm{~min}$. Hélio foi usado como gás de arraste, com fluxo de $1 \mathrm{~mL} / \mathrm{min}$.

Os espectros de massas foram obtidos através de análises dos extratos em um cromatógrafo a gás acoplado ao espectrômetro de massas (CG-EM), modelo Shimadzu QP- 2010 Plus, com ionização por impacto de elétrons $(70 \mathrm{eV})$. A coluna capilar e as condições de análises foram iguais às utilizadas nas análises de GC e a linha de transferência operou a $270^{\circ} \mathrm{C}$. Espectros de massas do banco de dados do Instituto Nacional de Padrões e Tecnologia (NIST27 e NIST147) foram utilizados no auxílio à identificação estrutural dos compostos encontrados nos extratos. A isomeria dos compostos que possuem insaturação foi definida através da coinjeção do extrato com padrões comerciais e sintéticos.

(E)-2-Hexenal, $(E)$-2-octenal, $(E)$-2-decenal e $(E)$-2-decen-1-ol foram comprados da Acros Organics (Geel, Turnhout, Belgium). Undecano, dodecano, tridecano, tetradecano, pentadecano, 1-trideceno, acetato de $(E)$-2-hexenila e acetato de $(E)$-2-octenila foram adquiridos da Aldrich Chemical Company (Milwaukee, Wisconsin, USA). (E)-4-Oxo-2-hexenal e (E)-4-oxo-2-octenal foram gentilmente cedidos pelo Dr. K. Chauhan, USDA-ARS Beltsville, MD, USA. ${ }^{10}$ Acetato de $(E)$-2-decenila foi preparado a partir do $(E)$-2-decen-1-ol. ${ }^{8}$

\section{Análises estatísticas}

Análises de variância (ANOVA) seguidas por teste Tukey ${ }^{11}$ foram utilizadas para comparar as porcentagens dos compostos presentes nos extratos das exúvias e das MTG.

\section{RESULTADOS E DISCUSSÃO}

No processo de troca de instar, as ninfas de pentatomídeos transferem o conteúdo de suas DAGs para as exúvias (Figura 1S, material suplementar). Nas análises cromatográficas realizadas com os extratos das exúvias, foi possível observar um total de 9 compostos para a espécie $L$. deducta e 8 compostos para $P$. stictica (Figura 1).

A partir de comparações com espectros de massa do banco de dados NIST27 e NIST147, comparações dos índices de Kovats (KI) com a literatura ${ }^{12}$ e coinjeção com padrões sintéticos, os compostos foram identificados como (E)-2-hexenal (a), $(E)$-4-oxo-2-hexenal (b), (E)-2-octenal (c), undecano (d), (E)-4-oxo-2-octenal (e), dodecano (f), (E)-2-decenal (g), 1-trideceno (h) e tridecano (i).

Os aldeídos encontrados (a, c e g) já foram descritos em grandes concentrações para outras espécies de pentatomídeos e, além de serem relatados como compostos defensivos ${ }^{13}$ e feromônios de alarme, ${ }^{4,14}$ alguns deles podem ter a função de cairomônio. Como exemplo, o parasitoide Telenomus podisi utiliza o $(E)$-2-hexenal para encontrar seu hospedeiro favorito, Euchistus heros. ${ }^{3,15}$

Os compostos a, d, f e i são toxinas conhecidas, irritantes e repelentes, sendo liberados pelos percevejos quando são molestados. Este fato sugere que eles realmente atuam como defesa química nestas espécies, porém não é conhecida a interação destes vários compostos. Gunawardena ${ }^{16}$ relatou que $(E)$-2-hexenal e tridecano causam mais efeito repelente quando combinados do que individualmente. Entretanto, outros alcanos, quando combinados com (E)-2-hexenal, não causam um efeito repelente tão efetivo quanto o tridecano. ${ }^{17}$

Nas análises quantitativas dos extratos das exúvias da espécie L. deducta, as proporções dos compostos na mistura feromonal dos cinco instares apresentaram variações, como pode ser visto na Tabela 1 (os valores das proporções podem ser visualizados na Tabela $1 \mathrm{~S}$, material suplementar). Com o aumento da fase ninfal, as mudanças

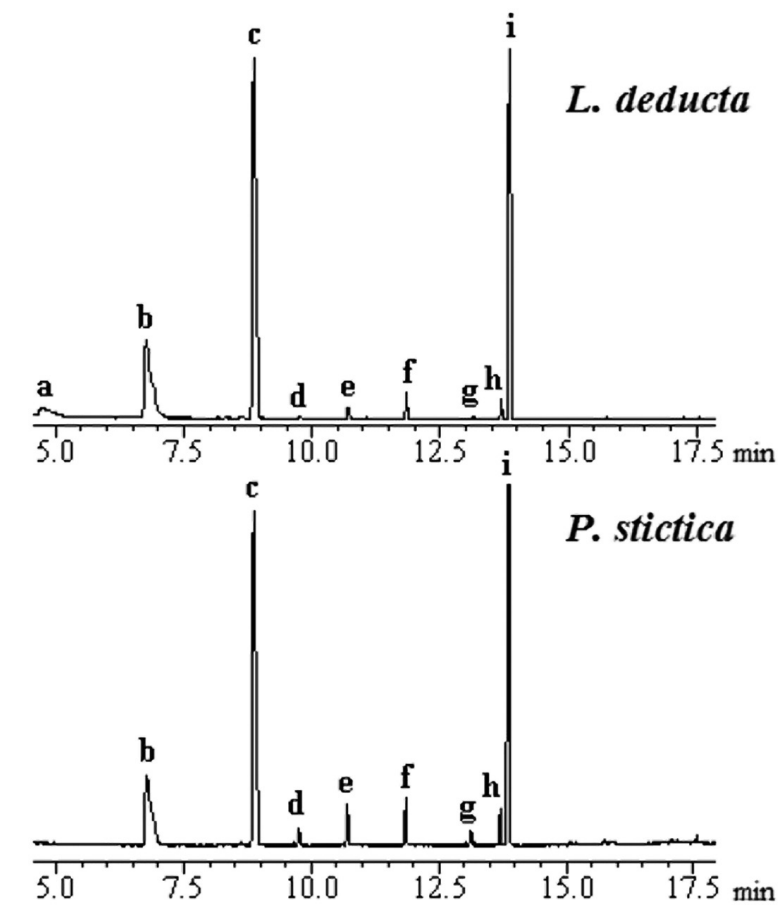

Figura 1. Exemplos de cromatogramas dos extratos das exúvias das espécies L. deducta e P. stictica

mais notórias foram o decréscimo nas proporções de tridecano (i), componente majoritário nos extratos de todos os instares, e $(E)-4$ oxo-2-octenal (e), e o aumento principalmente do (E)-2-octenal (c). (E)-4-Oxo-2-octenal (e) teve sua maior proporção no $1^{\circ}$ instar, estágio em que é possível observar um evidente comportamento de agregação, corroborando com outros estudos descritos por Fucarino et al..$^{18}$ que apontam outros oxo-alcenais como mediadores do comportamento de agregação em pentatomídeos.

Nos resultados quantitativos da espécie $P$. stictica todos os extratos dos cinco instares são compostos principalmente por tridecano (i), componente majoritário, (E)-2-octenal (c), composto que apresenta a segunda maior proporção, e (E)-4-oxo-2-hexenal (b) (Tabela $2 \mathrm{~S}$, material suplementar). Também fazem parte dessa mistura feromonal, pequenas proporções de $(E)$-4-oxo-2-octenal (e), dodecano (f) e 1-trideceno (h), somado a traços de undecano (d) e (E)-2-decenal (g).

Foi possível observar grandes variações nas proporções dos compostos com o aumento do instar, principalmente para aqueles que aparecem em maiores concentrações. Entretanto, como pode ser visto na Tabela 1, para esta espécie não foi possível estabelecer um padrão de variação, pois as proporções oscilaram muito. Como exemplo, o tridecano (10) aumentou sua proporção do primeiro para o segundo instar e a partir do terceiro instar voltou a diminuir.

Os percevejos adultos apresentam como fonte de seus compostos defensivos uma glândula grande, bem desenvolvida e colorida, a glândula metatorácica (MTG) (Figura 2S, material suplementar). Os resultados obtidos pela análise do extrato das MTGs das duas espécies apresentaram os mesmos compostos encontrados nas ninfas, com exceção do (E)-4-oxo-2-octenal (e), além do aparecimento de sete novos componentes (Figura 2). Esta similaridade entre ninfas e adultos é explicada por Carayon,,$^{19}$ que afirma que a glândula metatorácica de adultos de Hemiptera é morfologica e funcionalmente análoga ao terceiro par de glândulas abdominais dorsais das ninfas.

Os sete novos compostos foram identificados, utilizando-se a mesma metodologia dos compostos anteriores, como acetato de (E)-2-hexenila (j), (E)-2-nonenal (k), acetato de (E)-2-octenila (l), 
Tabela 1. Variação dos compostos encontrados no conteúdo das DAGs de ninfas de $1^{\circ}$ ao $5^{\circ}$ instar das espécies $L$. deducta e P. stictica

\begin{tabular}{|c|c|c|c|c|c|}
\hline & \multirow{2}{*}{ Compostos } & \multicolumn{2}{|c|}{ L. deducta } & \multicolumn{2}{|c|}{ P. stictica } \\
\hline & & Significância & Variação & Significância & Variação \\
\hline $\mathbf{a}$ & (E)-2-Hexenal & ns & & & - \\
\hline b & (E)-4-Oxo-2-hexenal & ns & & ns & \\
\hline c & (E)-2-Octenal & $*$ & & ns & \\
\hline d & Undecano & $\mathrm{ns}$ & & ns & \\
\hline e & (E)-4-Oxo-2-octenal & $*$ & & ns & \\
\hline f & Dodecano & ns & & ns & \\
\hline g & (E)-2-Decenal & ns & & * & \\
\hline $\mathbf{h}$ & 1-Trideceno & ns & & * & \\
\hline $\mathbf{i}$ & Tridecano & $*$ & & ns & \\
\hline
\end{tabular}

*Valores que apresentaram diferenças estatísticas entre médias, teste de Tukey $(\mathrm{p}<0,05)$; ns = diferença não significativa.

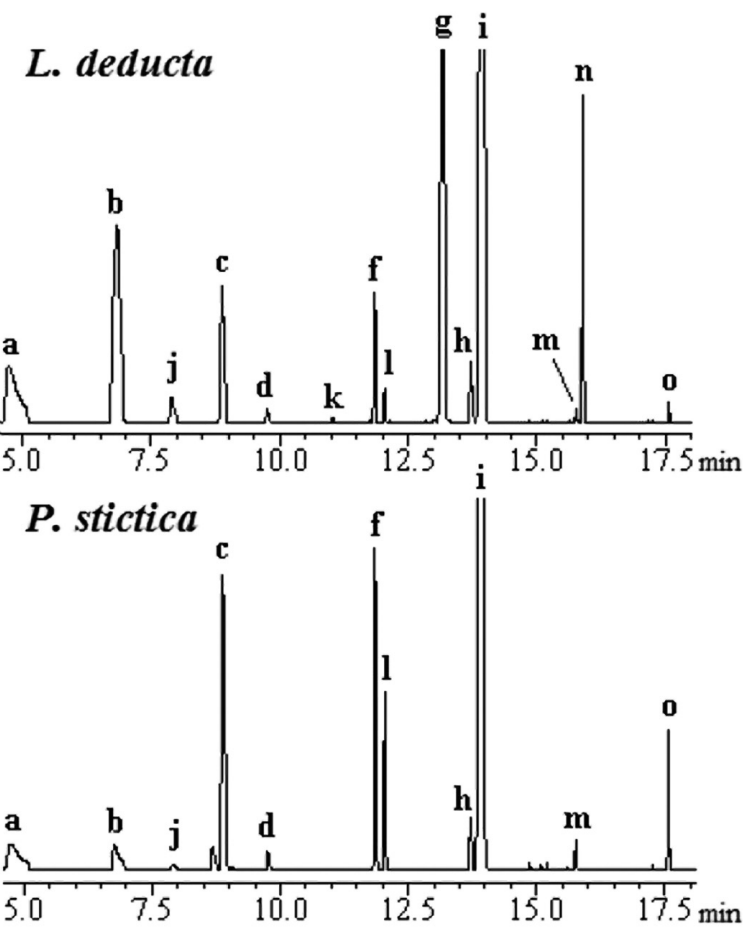

Figura 2. Cromatogramas dos extratos das glândulas metatorácicas de fêmeas com 10 dias de idade adulta das espécies L. deducta e P. stictica

tetradecano $(\mathbf{m})$, acetato de $(E)$-2-decenila $(\mathbf{n})$ e pentadecano $(\mathbf{o})$. Todas as estruturas químicas identificadas podem ser visualizadas na Figura 3 e a comparação de seus respectivos índices de Kovats com os descritos na literatura, na Tabela 2.

Segundo Aldrich et al., ${ }^{7}$ os ésteres são produzidos nas glândulas laterais da MTG e liberados para o reservatório para armazenamento, onde são convertidos a aldeídos através de hidrólise enzimática e oxidação alcoólica, justificando o aparecimento deste grupo apenas na mistura feromonal de insetos adultos. Ainda que encontrados em
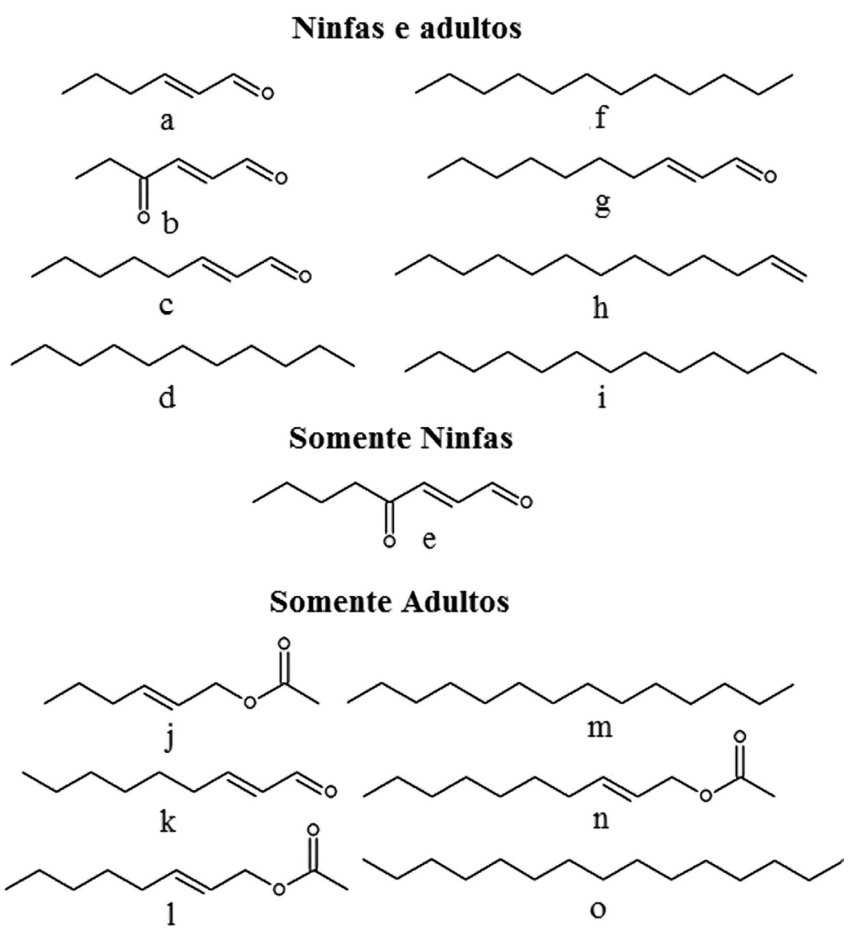

Figura 3. Estruturas químicas dos compostos presentes nas DAGs das ninfas e na MTG dos adultos das espécies L. deducta e P. stictica. a: (E)-2-hexenal, b: (E)-4-oxo-2-hexenal, $\boldsymbol{c}:(E)$-2-octenal, $\boldsymbol{d}$ : undecano, $\boldsymbol{e}:(E)-4-o x o-2-o c t e n a l, f$ : dodecano, $\boldsymbol{g}:(E)-2$-decenal, $\boldsymbol{h}: 1$-trideceno, $\boldsymbol{i}$ : tridecano, $\boldsymbol{j}:$ acetato de (E)-2-hexenila, $\boldsymbol{k}$ : (E)-2-nonenal, $\boldsymbol{l}$ : acetato de (E)-2-octenila, $\boldsymbol{m}$ : tetradecano, $\boldsymbol{n}$ : acetato de (E)-2-decenila, $\boldsymbol{o}$ : pentadecano

menor concentração do que aldeídos e hidrocarbonetos, os ésteres também têm mostrado importante função biológica como feromônio de alarme, como descrito para a espécie $N$. viridula. ${ }^{14}$

A composição do feromônio de defesa dos adultos da espécie 
L. deducta é formada principalmente por hidrocarbonetos, aldeídos, ésteres e oxo-acenal. $\mathrm{O}$ composto majoritário para ambos os sexos e em todas as idades estudadas é o tridecano (i), seguido por elevadas

Tabela 2. Tempos de retenção $\left(t_{R}\right)$ e comparação dos índices de Kovats (KI) calculados para os compostos presentes no conteúdo das DAGs de ninfas e MTG de adultos das espécies $L$. deducta e P. stictica, com os da ref. 12

\begin{tabular}{ccccc}
\hline & Compostos & $\begin{array}{c}\mathrm{t}_{\mathrm{R}} \\
(\mathrm{min})\end{array}$ & $\begin{array}{c}\mathrm{KI} \\
\text { natural }\end{array}$ & $\begin{array}{c}\mathrm{KI} \\
\text { literatura }\end{array}$ \\
\hline $\mathrm{a}$ & $(E)$-2-Hexenal & 4,7 & 858 & 855 \\
$\mathrm{~b}$ & $(E)$-4-Oxo-2-hexenal & 6,8 & 955 & -- \\
$\mathrm{c}$ & $(E)$-2-Octenal & 8,9 & 1055 & 1054 \\
$\mathrm{~d}$ & Undecano & 9,7 & 1097 & 1100 \\
$\mathrm{e}$ & $(E)$-4-Oxo-2-octenal & 10,7 & 1043 & -- \\
$\mathrm{f}$ & Dodecano & 11,8 & 1198 & 1200 \\
$\mathrm{~g}$ & $(E)$-2-Decenal & 13,1 & 1260 & 1263 \\
$\mathrm{~h}$ & 1-Trideceno & 13,7 & 1288 & 1291 \\
$\mathrm{i}$ & Tridecano & 13,9 & 1296 & 1300 \\
$\mathrm{j}$ & Acetato de $(E)-2-$-hexenila & 7,9 & 1010 & 1013 \\
$\mathrm{k}$ & $(E)$-2-Nonenal & 11,0 & 1159 & 1161 \\
$\mathrm{l}$ & Acetato de $(E)$-2-octenila & 12,0 & 1209 & 1209 \\
$\mathrm{~m}$ & Tetradecano & 15,8 & 1399 & 1400 \\
$\mathrm{n}$ & Acetato de $(E)$-2-decenila & 15,9 & 1407 & 1408 \\
$\mathrm{o}$ & Pentadecano & 17,6 & 1498 & 1500 \\
\hline
\end{tabular}

proporções de (E)-2-decenal (g) e (E)-4-oxo-2-hexenal (b) (Tabela $3 \mathrm{~S}$, material suplementar).

O conteúdo da glândula metatorácica dos machos não apresentou variação significativa na proporção dos compostos em comparação à glândula das fêmeas. Entretanto, houve diferenças nas proporções de um mesmo composto, quando comparadas glândulas de diferentes idades (10, 20 e 30 dias) da fase adulta. Na Tabela 3, é possível observar que ocorre o decréscimo da proporção do $(E)$-2-hexenal (a) e dos acetatos (j, l e n) após os 10 dias, enquanto que, inversamente, ocorre o aumento da proporção do (E)-2-decenal (g).

Os resultados obtidos para a espécie $P$. stictica também não apresentaram diferenças significativas nas proporções em que os compostos se encontram no conteúdo da MTG entre macho e fêmeas, mas esta diferença pode ser encontrada comparando-se as três idades estudadas, 10, 20 e 30 dias (Tabela 3). O tridecano (i) aparece novamente como componente majoritário do conteúdo das glândulas metatorácica, somado a concentrações significativas de (E)-2-octenal (c) (Tabela 4S, material suplementar). Todos os outros compostos estão presentes em pequenas proporções. Dessa forma, as variações significativas nas proporções dos componentes da mistura feromonal são vistas para o 1-trideceno (h), que aumenta de 10 para 20 dias e volta a diminuir em 30 dias, e para o acetato de $(E)$-2-octenila (l), que diminui após 10 dias.

As análises do conteúdo das glândulas produtoras dos compostos defensivos, tanto para as ninfas (DAG) quanto para os adultos (MTG) das espécies $L$. deducta e $P$. stictica, mostraram resultados compatíveis

Tabela 3. Variação dos compostos presentes nos extratos da MTG de adultos com 10, 20 e 30 dias das espécies L. deducta e P. stictica

\begin{tabular}{|c|c|c|c|c|c|}
\hline & \multirow{2}{*}{ Compostos } & \multicolumn{2}{|c|}{ L. deducta } & \multicolumn{2}{|c|}{ P. stictica } \\
\hline & & $\mathrm{p}$ & Variação & $\mathrm{p}$ & Variação \\
\hline $\mathbf{a}$ & (E)-2-Hexenal & $*$ & & ns & \\
\hline $\mathbf{b}$ & (E)-4-Oxo-2-hexenal & ns & & ns & \\
\hline c & (E)-2-Octenal & ns & & ns & \\
\hline d & Undecano & ns & & ns & \\
\hline $\mathbf{f}$ & Dodecano & ns & & ns & \\
\hline g & (E)-2-Decenal & $*$ & & & \\
\hline $\mathbf{h}$ & 1-Trideceno & ns & & $*$ & \\
\hline i & Tridecano & ns & & ns & \\
\hline $\mathbf{j}$ & Acetato de $(E)$-2-hexenila & $*$ & & ns & \\
\hline $\mathbf{k}$ & (E)-2-Nonenal & ns & & & \\
\hline $\mathbf{l}$ & Acetato de $(E)$-2-octenila & $*$ & & $*$ & \\
\hline m & Tetradecano & ns & & ns & \\
\hline $\mathbf{n}$ & Acetato de $(E)$-2-decenila & $*$ & & & \\
\hline $\mathbf{o}$ & Pentadecano & ns & & ns & \\
\hline
\end{tabular}

*Valores que apresentaram diferenças estatísticas entre médias, teste de Tukey $(\mathrm{p}<0,05)$; ns = diferença não significativa. 
com resultados já descritos na literatura para outras espécies de pentatomídeos. Na maioria destes estudos, o tridecano aparece como composto majoritário seguido de compostos pertencentes aos grupos dos aldeídos, oxo-alcenais, ésteres, entre outros. ${ }^{3}$

Entretanto, estudos relacionando a idade do inseto adulto à composição da mistura feromonal em pentatomídeos apenas foram relatados, anteriormente, por nosso grupo para a espécie $P$. macunai$m a .{ }^{8}$ Foi possível observar diferenças quantitativas na composição do feromônio de alarme destas espécies entre as diferentes idades adultas estudadas, provavelmente relacionadas ao amadurecimento do organismo destes insetos. Isto porque, segundo estudos realizados em nosso laboratório (dados ainda não publicados), estes insetos ainda não são sexualmente maduros com 10 dias de idade adulta e as principais diferenças estão entre essa idade em relação às demais (20 e 30 dias). Com estes dados, podemos prever que a MTG para os pentatomídeos também necessita de alguns dias para se formar completamente, já que esta só está presente na fase adulta.

Com este trabalho, corroborando com resultados obtidos para P. macunaima ${ }^{8}$ enfatizamos a necessidade da descrição da idade adulta em estudos com a MTG, dado que não apresentava relevante importância até então.

\section{MATERIAL SUPLEMENTAR}

As Figuras (1S e 2S) e as Tabelas (1S, 2S, 3S e 4S) estão disponíveis gratuitamente em http://quimicanova.sbq.org.br, na forma de arquivo PDF.

\section{AGRADECIMENTOS}

À Embrapa Soja, por possibilitar o acesso aos seus campos e ajuda na coleta de insetos, e ao suporte financeiro provido pela Coordenação de Aperfeiçoamento de Pessoal de Nível Superior (CAPES), Conselho Nacional de Desenvolvimento Científico e Tecnológico (CNPq) e pelo Instituto Nacional de Ciências e Tecnologia de Semioquímicos na Agricultura (INCT)

\section{REFERÊNCIAS}

1. Aldrich, J. R.; Annu. Rev. Entomol. 1988, 33, 211.

2. Borges, M.; Aldrich, J. R.; Experientia 1992, 48, 893.

3. Moraes, M. C. B.; Pareja, M.; Laumann, R. A.; Borges, M.; Neotrop. Entomol. 2008, 37, 489.

4. Pavis, C.; Malosse, C.; Ducrot, P. H.; Descoins, C.; J. Chem. Ecol. 1994, $20,2213$.

5. Ho, H.-Y.; Millar, J. G.; Zool. Stud. 2001, 40, 6.

6. Pareja, M.; Borges, M.; Laumann, R. A.; Moraes, M. C. B.; J. Insect Physiol. 2007, 53, 639.

7. Aldrich, J. R.; Blum, M. S.; Hefetz, A.; Fales, H. M.; Lloyd, H. A.; Roller, P.; Science 1978, 201, 452.

8. Fávaro, C. F.; Rodrigues, M. A. C. de M.; Aldrich, J. R.; Zarbin, P. H. G.; J. Braz. Chem. Soc. 2011, 22, 58.

9. Siqueira, F.; Dissertação de Mestrado, Universidade Federal do Paraná, Brasil, 2007.

10. Feldlaufer, M. F.; Domingue, M. J.; Chauhan, K. R.; Aldrich, J. R.; J. Med. Entomol. 2010, 47, 140.

11. Ayres, M.; Ayres, M. J.; Ayres, D. L.; Santos, A. S.; BioEstat3.0, Aplicações estatísticas nas áreas das ciências biológicas e médicas, Sociedade Civil Mamirauá: Belém, 2003.

12. Adams, R. P.; Identification of essential oil components by gas chromatography - mass spectrometry, $4^{\text {th }}$ ed., Allured Publ. Corporation: Illinois, 2007.

13. Gilby, A. R.; Waterhouse, D. F.; Nature 1967, 216, 90.

14. Lockwood, J. A.; Story, R. N.; Ann. Entomol. Soc. Am. 1987, 80, 686

15. Peres, W. A. A.; Tese de Doutorado, Universidade Federal do Paraná, Brasil, 2004.

16. Gunawardena, N. E.; Herath, H. M. W. K. B.; J. Chem. Ecol. 1991, 17, 2449.

17. Zarbin, P. H. G.; Borges, M.; dos Santos, A. A.; de Oliveria, A. R. M.; Simonelli, F.; Marques, F. A.; J. Braz. Chem. Soc. 2000, 11, 424.

18. Fucarino, A.; Millar, J. G.; McElfresh, J. S.; Colazza, S.; J. Chem. Ecol. 2004, 30, 1257

19. Carayon, J.; Ann. Soc. Entomol. Fr. 1971, 7, 737. 


\section{IDENTIFICAÇÃO DOS COMPOSTOS DEFENSIVOS ENCONTRADOS NAS GLÂNDULAS METATORÁCICA E} ABDOMINAIS DORSAIS DOS PERCEVEJOS Loxa deducta E Pellaea stictica (Heteroptera: Pentatomidae)

Carla F. Fávaro e Paulo H. G. Zarbin*

Departamento de Química, Universidade Federal do Paraná, CP 19081, 81531-990 Curitiba - PR, Brasil
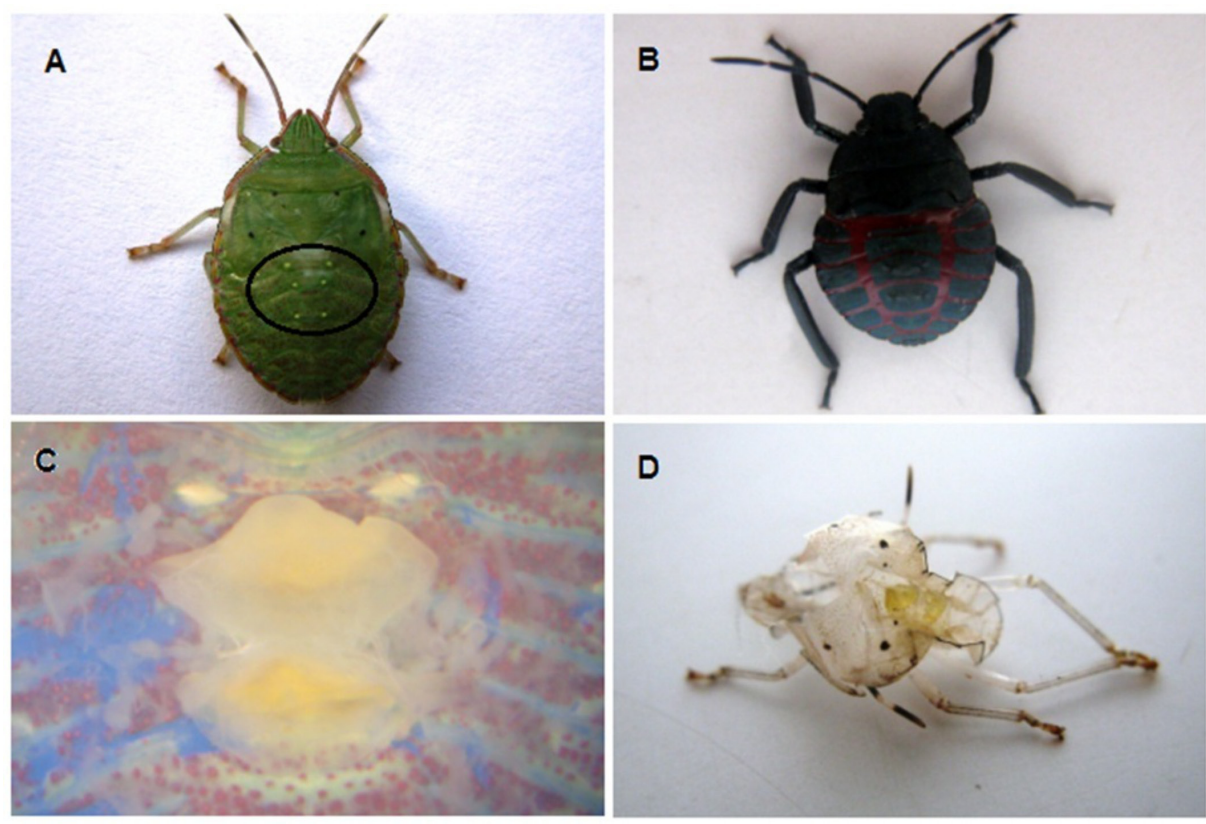

Figura 1S. A) Localização da glândula abdominal dorsal em uma ninfa da espécie L. deducta, B) ninfa da espécie P. stictica, C) glândula abdominal dorsal e D) exúvia liberada no processo de troca de instar da espécie L. deducta
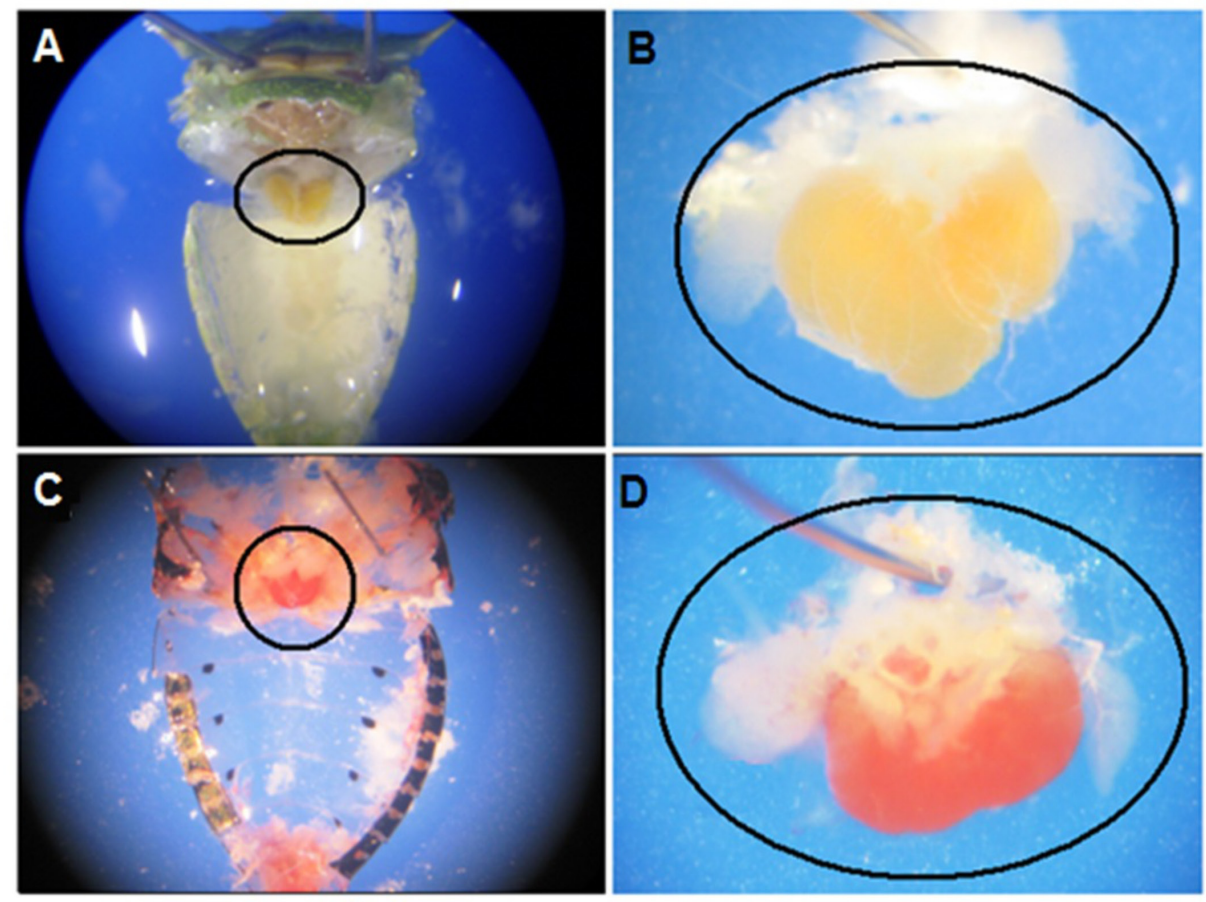

Figura 2S. A) MTG de fêmea de L. deducta, B) MTG extraída de L. deducta, C) MTG de fêmea de P. stictica e D) MTG extraída de P. stictica 
Tabela 1S. Porcentagem dos compostos encontrados nas glândulas abdominais dorsais das ninfas de $L$. deducta (média \pm desvio padrão) ( $\mathrm{n}=3$ ). Valores em \% por exúvia. Teste de Tukey p >0,05. Em negrito encontram-se os compostos que apresentaram diferenças significativas entre os instares

\begin{tabular}{|c|c|c|c|c|c|c|}
\hline & Composto & $1^{\circ}$ ínstar & $2^{\circ}$ ínstar & $3^{\circ}$ ínstar & $4^{\circ}$ ínstar & $5^{\circ}$ ínstar \\
\hline $\mathrm{a}$ & (E)-2-Hexenal & $0 \mathrm{a}$ & $0,6 \pm 0,7 \mathrm{a}$ & $1,3 \pm 1,2 \mathrm{a}$ & $0,4 \pm 0,3 \mathrm{a}$ & $1,3 \pm 1,7 \mathrm{a}$ \\
\hline $\mathrm{b}$ & (E)-4-Oxo-2-hexenal & $10,3 \pm 2,2 \mathrm{a}$ & $10,0 \pm 1,6 \mathrm{a}$ & $15,3 \pm 1,2 \mathrm{a}$ & $6,9 \pm 2,0 \mathrm{a}$ & $12,3 \pm 4,0 \mathrm{a}$ \\
\hline c & (E)-2-Octenal & $25,3 \pm 0,7 a$ & $25,9 \pm 2,9 \mathrm{a}$ & $32,0 \pm 1,4 a b$ & $34,0 \pm 1,4 b$ & $38,9 \pm 5,8 b$ \\
\hline d & Undecano & $0 \mathrm{a}$ & $0,3 \pm 0,1 \mathrm{a}$ & $0,2 \pm 0,0 \mathrm{a}$ & $0,9 \pm 0,1 \mathrm{a}$ & $0,7 \pm 0,7 \mathrm{a}$ \\
\hline $\mathbf{e}$ & (E)-4-Oxo-2-octenal & $4,0 \pm 1,8 a$ & $0,8 \pm 0,3 b$ & $1,0 \pm 0,3 b$ & $0,8 \pm 0,1 b$ & $1,5 \pm 0,2 \mathrm{ab}$ \\
\hline $\mathrm{f}$ & Dodecano & $1,6 \pm 0,2 \mathrm{a}$ & $1,4 \pm 0,2 \mathrm{a}$ & $1,6 \pm 0,6 \mathrm{a}$ & $2,7 \pm 0,3 \mathrm{a}$ & $1,8 \pm 0,9 \mathrm{a}$ \\
\hline $\mathrm{g}$ & (E)-2-Decenal & $0 \mathrm{a}$ & $0,2 \pm 0,0 \mathrm{a}$ & $0,1 \pm 0,0 \mathrm{a}$ & $0,4 \pm 0,1 \mathrm{a}$ & $0,3 \pm 0,3 \mathrm{a}$ \\
\hline $\mathrm{h}$ & 1-Trideceno & $0,90 \pm 0,3 \mathrm{a}$ & $0,6 \pm 0,1 \mathrm{a}$ & $0,6 \pm 0,1 \mathrm{a}$ & $2,2 \pm 0,3 \mathrm{a}$ & $1,2 \pm 1,0 \mathrm{a}$ \\
\hline $\mathbf{i}$ & Tridecano & $58,0 \pm 3,9 a$ & $60,2 \pm 4,9$ a & $48,0 \pm 2,1 a b$ & $51,7 \pm 3,5 \mathrm{ab}$ & $42,1 \pm 5,0 b$ \\
\hline
\end{tabular}

Tabela 2S. Porcentagem dos compostos encontrados nas glândulas abdominais dorsais das ninfas de $P$. stictica (média \pm desvio padrão) ( $\mathrm{n}=3$ ). Valores em \% por exúvia. Teste de Tukey p >0,05. Em negrito encontram-se os compostos que apresentaram diferenças significativas entre os instares

\begin{tabular}{ccccccc}
\hline & Composto & $1^{\circ}$ ínstar & $2^{\circ}$ ínstar & $3^{\circ}$ ínstar & $4^{\circ}$ ínstar & $5^{\circ}$ ínstar \\
\hline b & $(E)$-4-Oxo-2-hexenal & $13,9 \pm 4,8 \mathrm{a}$ & $3,3 \pm 2,7 \mathrm{a}$ & $5,6 \pm 3,2 \mathrm{a}$ & $5,2 \pm 4,5 \mathrm{a}$ & $4,7 \pm 2,0 \mathrm{a}$ \\
$\mathrm{c}$ & $(E)$-2-Octenal & $32,3 \pm 1,4 \mathrm{a}$ & $17,7 \pm 1,9 \mathrm{a}$ & $17,7 \pm 3,8 \mathrm{a}$ & $22,1 \pm 9,3 \mathrm{a}$ & $32,0 \pm 8,4 \mathrm{a}$ \\
$\mathrm{d}$ & Undecano & $0 \mathrm{a}$ & $0,2 \pm 0,0 \mathrm{a}$ & $0,2 \pm 0,1 \mathrm{a}$ & $0,4 \pm 0,3 \mathrm{a}$ & $0,5 \pm 0,2 \mathrm{a}$ \\
$\mathrm{e}$ & $(E)-4$-Oxo-2-octenal & $2,9 \pm 2,3 \mathrm{a}$ & $0,5 \pm 0,1 \mathrm{a}$ & $1,0 \pm 0,7 \mathrm{a}$ & $0,5 \pm 0,2 \mathrm{a}$ & $0,7 \pm 0,3 \mathrm{a}$ \\
$\mathrm{f}$ & Dodecano & $1,3 \pm 0,4 \mathrm{a}$ & $1,7 \pm 0,4 \mathrm{a}$ & $1,6 \pm 0,2 \mathrm{a}$ & $2,1 \pm 0,2 \mathrm{a}$ & $2,0 \pm 0,4 \mathrm{a}$ \\
g & $(\boldsymbol{E})$-2-Decenal & $\mathbf{1 , 1} \pm \mathbf{0 , 2 a}$ & $\mathbf{0 , 1} \pm \mathbf{0 , 0 b}$ & $\mathbf{0 , 1} \pm \mathbf{0 , 0 b}$ & $\mathbf{0 , 2} \pm \mathbf{0 , 1 b}$ & $\mathbf{0 , 1} \pm \mathbf{0 , 0 b}$ \\
h & $\mathbf{1 - T r i d e c e n o}$ & $\mathbf{0 , 6} \pm \mathbf{0 , 2 a}$ & $\mathbf{1 , 3} \pm \mathbf{0 , 1} \mathbf{a b}$ & $\mathbf{1 , 3} \pm \mathbf{0 , 3 a b}$ & $\mathbf{2 , 4} \pm \mathbf{0 , 8 a b}$ & $\mathbf{4 , 0} \pm \mathbf{1 , 7 b}$ \\
$\mathrm{i}$ & Tridecano & $48,0 \pm 8,8 \mathrm{a}$ & $75,2 \pm 4,2 \mathrm{a}$ & $72,4 \pm 7,1 \mathrm{a}$ & $67,3 \pm 15,2 \mathrm{a}$ & $55,7 \pm 10,2 \mathrm{a}$ \\
\hline
\end{tabular}

Tabela 3S. Porcentagem dos compostos encontrados nas glândulas metatorácicas (MTG) dos adultos de L. deducta (média \pm desvio padrão) ( $\mathrm{n}=5$ ). Valores em \% por MTG. Teste de Tukey p > 0,05. Em negrito encontram-se os compostos que apresentam diferenças significativas em suas proporções nas diferentes idades estudadas

\begin{tabular}{|c|c|c|c|c|}
\hline & Composto & 10 dias & 20 dias & 30 dias \\
\hline $\mathbf{a}$ & $(E)$-2-Hexenal & $7,7 \pm 1,8 \mathrm{a}$ & $2,9 \pm 1,7 b$ & $1,6 \pm 0,6 b$ \\
\hline $\mathrm{b}$ & (E)-4-Oxo-2-hexenal & $10,7 \pm 5,6 \mathrm{a}$ & $11,5 \pm 8,7 \mathrm{a}$ & $11,6 \pm 8,7 \mathrm{a}$ \\
\hline $\mathrm{c}$ & (E)-2-Octenal & $3,6 \pm 1,4 \mathrm{a}$ & $3,7 \pm 1,6 \mathrm{a}$ & $3,0 \pm 1,0 \mathrm{a}$ \\
\hline $\mathrm{d}$ & Undecano & $0,2 \pm 0,1 \mathrm{a}$ & $0,3 \pm 0,1 \mathrm{a}$ & $0,3 \pm 0,2 \mathrm{a}$ \\
\hline $\mathrm{f}$ & Dodecano & $2,0 \pm 0,5 \mathrm{a}$ & $2,5 \pm 0,8 \mathrm{a}$ & $2,4 \pm 0,8 \mathrm{a}$ \\
\hline $\mathbf{g}$ & $(E)$-2-Decenal & $19,7 \pm 4,2 \mathrm{a}$ & $24,0 \pm 5,7 a b$ & $29,1 \pm 4,0 b$ \\
\hline $\mathrm{h}$ & 1-Trideceno & $0,8 \pm 0,4 \mathrm{a}$ & $0,9 \pm 0,3 \mathrm{a}$ & $0,6 \pm 0,2 \mathrm{a}$ \\
\hline $\mathrm{i}$ & Tridecano & $44,5 \pm 8,3 \mathrm{a}$ & $51,6 \pm 15,7 \mathrm{a}$ & $48,4 \pm 12,5 \mathrm{a}$ \\
\hline $\mathbf{j}$ & Acetato de $(E)$-2-hexenila & $3,1 \pm 3,0 \mathrm{a}$ & $0,5 \pm 0,4 b$ & $0,4 \pm 0,4 b$ \\
\hline $\mathrm{k}$ & (E)-2-Nonenal & $0,1 \pm 0,0 \mathrm{a}$ & $0,1 \pm 0,1 \mathrm{a}$ & $0,1 \pm 0,0 \mathrm{a}$ \\
\hline 1 & Acetato de $(E)$-2-octenila & $0,9 \pm 0,8 a$ & $0,2 \pm 0,1 b$ & $0,1 \pm 0,1 b$ \\
\hline $\mathrm{m}$ & Tetradecano & $0,2 \pm 0,1 \mathrm{a}$ & $0,7 \pm 0,2 \mathrm{a}$ & $0,4 \pm 0,3 \mathrm{a}$ \\
\hline $\mathbf{n}$ & Acetato de $(E)$-2-decenila & $6,7 \pm 4,9 \mathrm{a}$ & $1,3 \pm 0,6 b$ & $1,6 \pm 1,0 b$ \\
\hline o & Pentadecano & $0,3 \pm 0,1 \mathrm{a}$ & $0,4 \pm 0,2 \mathrm{a}$ & $0,4 \pm 0,2 \mathrm{a}$ \\
\hline
\end{tabular}


Tabela 4S. Porcentagem dos compostos encontrados nas glândulas metatorácicas $(\mathrm{MTG})$ dos adultos de $P$. stictica (média \pm desvio padrão) (n = 5). Valores em \% por MTG. Teste de Tukey p $>0,05$. Em negrito encontram-se os compostos que apresentam diferenças significativas em suas proporções nas diferentes idades estudadas

\begin{tabular}{|c|c|c|c|c|}
\hline & Composto & 10 dias & 20 dias & 30 dias \\
\hline a & (E)-2-Hexenal & $1,2 \pm 0,7 \mathrm{a}$ & $2,3 \pm 1,7 \mathrm{a}$ & $1,5 \pm 1,5 \mathrm{a}$ \\
\hline $\mathrm{b}$ & (E)-4-Oxo-2-hexenal & $0,2 \pm 0,3 \mathrm{a}$ & $2,3 \pm 1,5 \mathrm{a}$ & $2,4 \pm 2,7 \mathrm{a}$ \\
\hline $\mathrm{c}$ & (E)-2-Octenal & $6,0 \pm 1,7 \mathrm{a}$ & $11,0 \pm 4,4 a$ & $11,3 \pm 7,0 \mathrm{a}$ \\
\hline $\mathrm{d}$ & Undecano & $0,2 \pm 0,0 \mathrm{a}$ & $0,3 \pm 0,1 \mathrm{a}$ & $0,3 \pm 0,1 \mathrm{a}$ \\
\hline $\mathrm{f}$ & Dodecano & $2,7 \pm 0,6 \mathrm{a}$ & $3,3 \pm 0,8 \mathrm{a}$ & $3,3 \pm 1,0 \mathrm{a}$ \\
\hline $\mathbf{h}$ & 1-Trideceno & $0,7 \pm 0,1 a$ & $1,1 \pm 0,4 b$ & $0,7 \pm 0,2 \mathbf{a b}$ \\
\hline $\mathrm{i}$ & Tridecano & $86,6 \pm 3,1 \mathrm{a}$ & $77,1 \pm 5,8 \mathrm{a}$ & $77,6 \pm 9,1 \mathrm{a}$ \\
\hline $\mathrm{j}$ & Acetato de $(E)$-2-hexenila & $0,2 \pm 0,4 \mathrm{a}$ & $0,0 \pm 0,1 \mathrm{a}$ & $0,0 \pm 0,1 \mathrm{a}$ \\
\hline 1 & Acetato de $(E)$-2-octenila & $\mathbf{0 , 7} \pm \mathbf{0 , 5 a}$ & $0,2 \pm 0,2 b$ & $0,1 \pm 0,2 b$ \\
\hline $\mathrm{m}$ & Tetradecano & $0,2 \pm 0,1 \mathrm{a}$ & $0,3 \pm 0,1 \mathrm{a}$ & $0,3 \pm 0,1 \mathrm{a}$ \\
\hline o & Pentadecano & $0,9 \pm 0,3 \mathrm{a}$ & $1,4 \pm 0,6 \mathrm{a}$ & $1,7 \pm 1,0 \mathrm{a}$ \\
\hline
\end{tabular}

